\title{
Simulation and Analysis the Effect of Varying no. of Nodes on AODV and DSR for different Applications
}

\author{
Sumit Khurana \\ M Tech Student ECE \\ Shaheed Bhagat Singh State Technical Campus, \\ Ferozepur \\ Punjab 152004
}

\author{
Amit Grover \\ AP ECE \\ Shaheed Bhagat Singh State Technical Campus, \\ Ferozepur \\ Punjab 152004
}

\begin{abstract}
MANET (Mobile Ad-hoc network) is a decentralized and selforganizing network. At present trends because of its security provided by MANET, so MANET becomes one of the most important wireless communication mechanisms among all other. In MANET within the network, the intermediate nodes route the packets from the source node to the destination node without any centralization authority to do, so MANET used the various kinds of routing protocol and these routing protocols are AODV, DSR, GRP and OLSR etc. In this paper the performance of AODV and DSR is analyzed by varying no. of nodes for two different applications: video conferencing and VOIP. The given work will be analyzed using OPNET Modeler. The result is carryout via Delay, Retransmission Attempt and Throughput. The results show that the overall performance of AODV is better than DSR for both the applications.
\end{abstract}

\section{Keywords: MANET, AODV, DSR, OPNET}

\section{INTRODUCTION}

For short distance we use Bluetooth, Wi-Fi but long distance communication can be done by using MANET. In Mobile Adhoc network (MANET) wireless nodes to communicate with each other wirelessly over wide area without any central devices to provide peer to peer communication. So it is open and secure network [1]. MANET can be used in many fields like military battlefields, classrooms and rescue sites because it provides security. It can also be used in rapid development areas and where there is no wireline network available [2]. To allow communication between devices there are different types of protocols like AODV, DSR, GRP etc. One of the main areas of research is to find the protocol that performs best in different applications. In this Paper the performance of AODV and DSR routing protocols is evaluated by increasing the number of nodes using two applications video conferencing and VOIP. The rest of paper is organized as follows: next Section presents brief overview of MANET routing protocols that we evaluate. Then next section describes Related Work, and then simulation environment is described. Simulation results are shown in next Section followed by conclusion and future scope.

\section{ROUTING PROTOCOLS IN}

\section{MANET}

There are three categories of Routing Protocol in MANET: Active, Proactive and Hybrid [3][4]. This section describes the working of two protocols: - AODV (Ad Hoc On-Demand
Distance-Vector Routing Protocol) and DSR PROTOCOL (Dynamic Source Routing).

\subsection{AODV (Ad Hoc On-Demand Distance- Vector Routing Protocol)}

AODV provides route on demand. In AODV, when ever source wants to send data to destination they start route discovery process; in this source node sends a route request message to its neighbors. If neighbor has information about destination it sends route reply message to source node in unicast mode, if not then it sends a message to all of its neighbors and so on. This process will remain continue until the information about destination will not found. On the basis of this process a route called reverse path is recorded, which identify the path. By using this path route reply message is send back to the source node. When the source node receive route reply message the route becomes ready and the source node starts sending data packets $[5,6]$.

\subsection{DSR PROTOCOL (Dynamic Source Routing)}

Dynamic Source Routing (DSR) [7] is a reactive protocol i.e. It doesn't use periodic advertisements. It computes the routes when necessary and then maintains them. Source routing is a routing technique in which the sender of a packet determines the complete sequence of nodes through which the packet has to pass; the sender explicitly lists this route in the packet's header, identifying each forwarding "hop" by the address of the next node to which to transmit the packet on its way to the destination host. There are two significant stages in working of DSR: Route Discovery and Route Maintenance. A host initiating a route discovery broadcasts a route request packet which may be received by those hosts within wireless transmission range of it. The route request packet identifies the host, referred to as the target of the route discovery, for which the route is requested. If the route discovery is successful, the initiating host receives a route reply packet listing a sequence of network hops through which it may reach the target. While a host is using any source route, it monitors the continued correct operation of that route. This monitoring of the correct operation of a route in use is called route maintenance. When route maintenance detects a problem with a route in use, route discovery may be used again to discover a new, correct route to the destination. DSR Protocol also provides communication without creating any loop [8].

\section{RELATED WORK}

In 2013, Sumit Mahajan, Vinay Chopra [9] compare three routing protocols AODV, OLSR and TORA by using OPNET 
Modeler in terms of delay, network load and throughput, jitter, mean opinion score and concludes that performance of TORA is good as compare to OLSR and AODV.

In 2013, Gagangeet Singh Aujla et al [10] compare four routing protocols AODV, DSR, TORA and GRP in terms of throughput, delay, load and data dropped and concluded that the overall study of both the applications shows that AODV is best suited protocol for video conferencing for lower number of nodes and OLSR can be used as a replacement as its performance degrades for high number of nodes. The OLSR protocol shows best performance for email traffic but GRP is also equally good. GRP performance is better for low number of nodes but it degrades with increase in number of nodes but OLSR improves its performance. TORA shows poor results in both scenarios followed by DSR. In both scenarios GRP and AODV suits the low number of nodes whereas OLSR suits better for high number of nodes.

No one has compared the performance of AODV and DSR for Video Conferencing (High resolution video) and VOIP at $18 \mathrm{mbps}$ data rate. So the performance of AODV and DSR is evaluated for Video Conferencing (High resolution video) and VOIP at $18 \mathrm{mbps}$ Data rate.

\section{SIMULATION ENVIRONMENT}

In this experiment the effect of varying no. of nodes on AODV and DSR for two different applications is analyzed. For these three scenarios has been made. The first scenario has 100 nodes, the second scenario has 125 nodes and the last scenario has 150 nodes. In this experiment the placement of nodes is random over an area of $1000 \mathrm{~m} \times 1000 \mathrm{~m}$. The simulation is run for $300 \mathrm{~s}$ with a seed value of 128 . The mobility model used is "Random Waypoint Model". Random waypoint is most widely used mobility model in which a node randomly chooses a destination, called waypoint and moves towards it in a straight line with a constant velocity [11][12].

OPNET Simulator 14.5 [13] was used to analyze the performance of AODV and DSR protocol. We used OPNET modeler, as OPNET modeler provides a comprehensive development environment supporting the modeling of communication network and distributed systems [14] and it also supports both applications. OPNET modeler provides better environment for simulation, data collection and data analysis [13]. The Simulation parameters used in our scenario are shown in Table 1.

Table 1: Simulation Parameters

\begin{tabular}{|l|l|}
\hline $\begin{array}{l}\text { Network } \\
\text { Parameters }\end{array}$ & Values \\
\hline Number of Nodes & 100,125 and 150 \\
\hline Simulation Time & $300 \mathrm{sec}$ (5 min) \\
\hline Simulation Area & $1000 \mathrm{~m}$ X 1000 m \\
\hline Routing Protocols & AODV, DSR \\
\hline $\begin{array}{l}\text { Node Movement } \\
\text { Model }\end{array}$ & Random Waypoint \\
\hline Data Rate & $18 \mathrm{mbps}$ \\
\hline Application Name & $\begin{array}{l}\text { Video Conferencing (High resolution } \\
\text { video) and VOIP }\end{array}$ \\
\hline Simulator & Opnet Modeler 14.5 \\
\hline
\end{tabular}

\section{RESULTS}

The main target of this paper is to evaluate the performance of AODV and DSR protocols by varying the number of nodes for two different applications i.e. video conferencing and
VOIP. Performance is compared in terms of Delay, Retransmission Attempt and Throughput.

\subsection{Delay}

Fig 1 shows the result of delay of AODV and DSR for 100, 125 and 150 nodes for Video Conferencing application. From Fig. 1 it is clear that with increase in no. of nodes Delay decreases for both AODV and DSR. From Fig. 1 the result shows that with increase in number of nodes the performance of AODV is better than DSR. So AODV shows less delay with increase in number of users than DSR.



Figure 1 Delay for Video conferencing



Figure 2 Delay for VOIP

Fig 2 shows the result of AODV and DSR for VOIP applications. Result shows that the delay of AODV is less as compare to DSR. Fig 2 also shows that as we increase the no. of users, the delay also increases for AODV and DSR.

\subsection{Retransmission Attempt}

Fig 3 shows the result of Retransmission Attempt of AODV and DSR for 100, 125 and 150 nodes for Video Conferencing application. Fig 3 shows that with increase in no. of users 
retransmission attempt for AODV also increases but for DSR it increases for 125 nodes and then again decreases. The result shows that Retransmission of DSR is less than AODV.

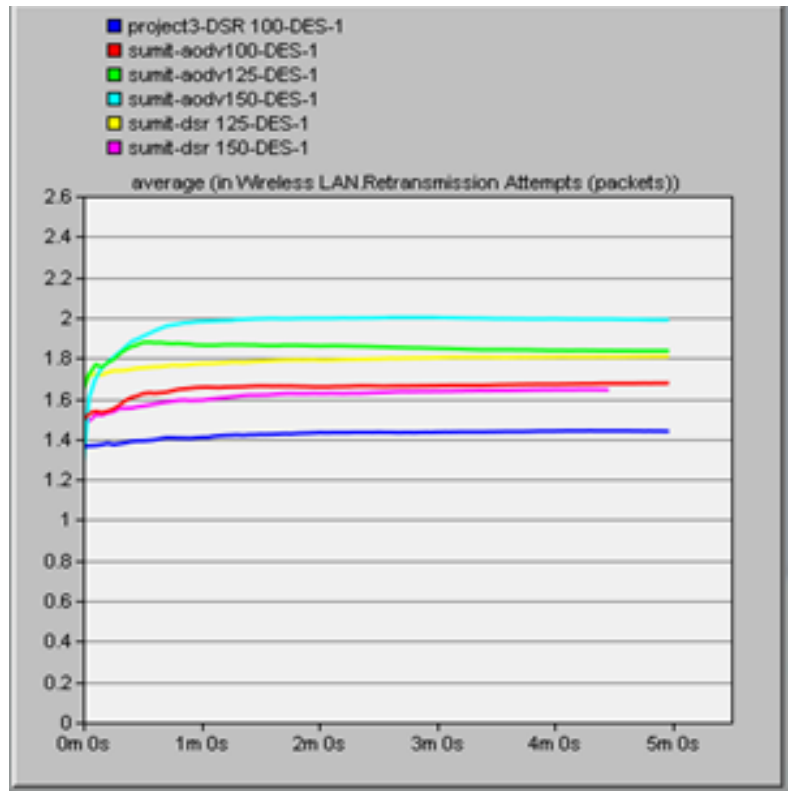

Figure 3 Retransmission Attempt for Video Conferencing



Figure 4 Retransmission Attempt for VOIP

Fig. 4 shows the result of Retransmission attempt for VOIP application. Result shows that as no. of user increases Retransmission attempt also increases. Fig. 4 also shows that the performance of DSR is slightly better than AODV.

\subsection{Throughput}

Fig 5 shows the result of Throughput of AODV and DSR for 100, 125 and 150 nodes for Video Conferencing application Fig. 5 shows that with increase in no. of node throughput of AODV and DSR is increases. Fig. 5 shows that the throughput of AODV is high than DSR.

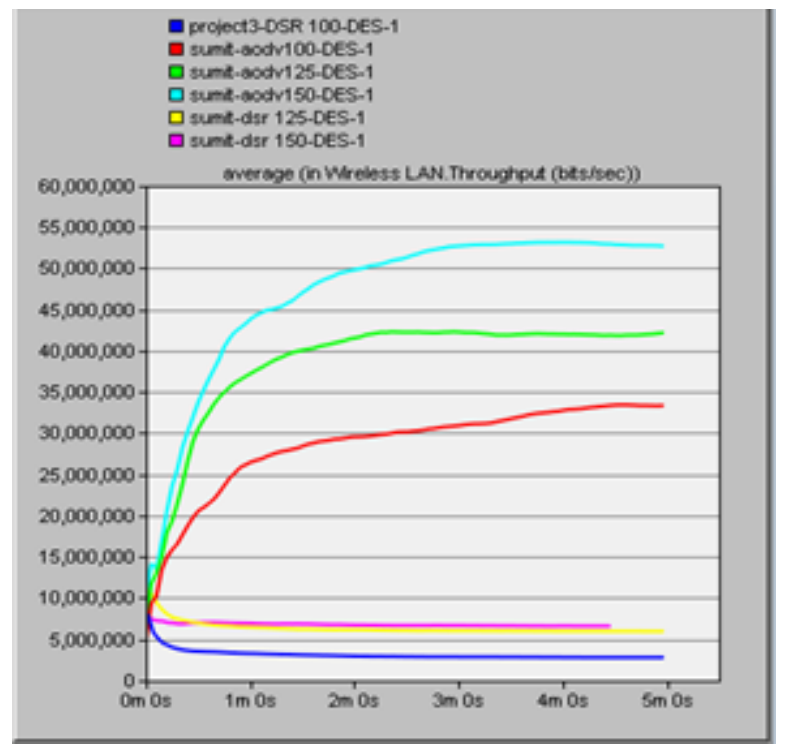

Figure 5 Throughput for Video Conferencing

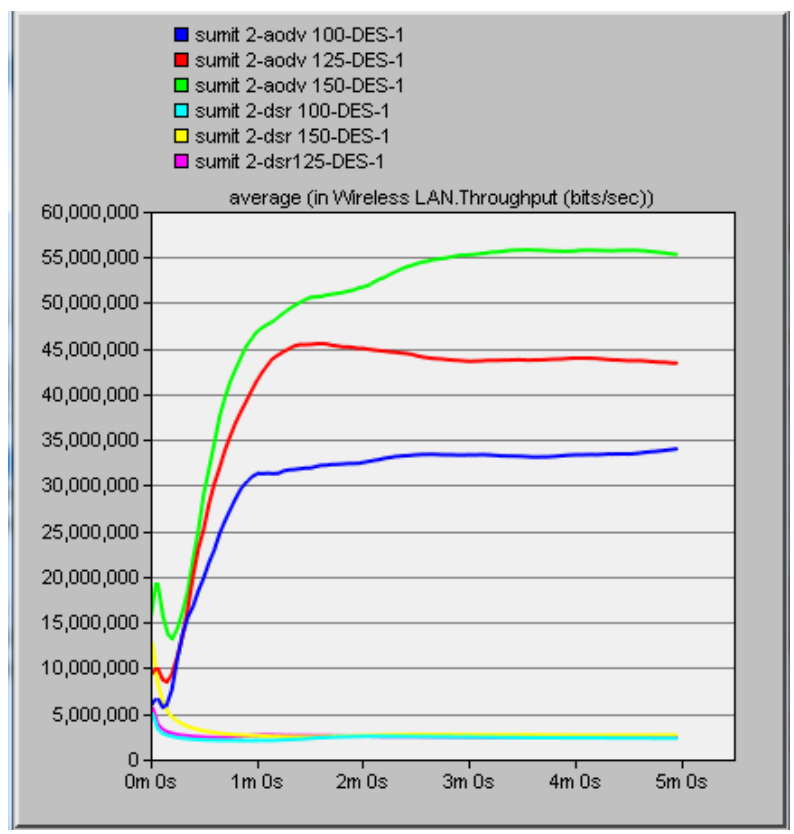

Figure 6 Throughput for VOIP

Fig. 6 shows the result of Throughput of AODV and DSR for the application of VOIP. The result shows that for AODV as we increase the no. of users the throughput increases but for DSR this change is very less. Fig 6 also shows that the performance of AODV is better than DSR.

\section{CONCLUSION}

In these research analyses the performance of AODV and DSR by varying no. of nodes is carried out for two different applications (video conferencing and VOIP) in terms of Delay Retransmission Attempt and Throughput. In this experiment the placement of nodes is random. Here each node is move with the speed of $5 \mathrm{~m} / \mathrm{s}$ in an area of within the network range $10,00,000 \mathrm{sq} \mathrm{m}$. The study of simulation shows that AODV has less Delay and high Retransmission Attempt and throughput for video conferencing and VOIP and DRS have high Delay and less Retransmission Attempt and throughput 
for video conferencing and VOIP. The simulation results show that the overall performance of ADOV is high than DSR as the no. of nodes increases. This work can be extend by considering some other important parameters like network size, number of nodes, which can further be useful to conduct study of these protocols under different network scenarios and configurations.

\section{REFERENCES}

[1] Kuldeep Vats et al, "Simulation and performance Analysis of OLSR, GRP, DSR Routing Protocol using OPNET" IJETED Volume 2, Issue 2, March 2012.

[2] T. Kunz and E. Cheng, "On-demand multicasting in adhoc networks: Comparing AODV and ODMRP,"pp. 453454, 2002.

[3] C. Tschudin, P. Gunningberg, H. Lundgren, and E. Nordstrom, "Lessons from Experimental MANET Research," Elsevier Ad Hoc Networks Journal, Vol. 3, Issue 3, March 2005.

[4] M. Conti and S. Giordana, "Multihop Ad Hoc Networking: The Reality," IEEE Communications Magazine, April 2007.

[5] C. Perkins, E. Belding-Royer, S. Das, quet,"Ad hoc OnDemand Distance Vector (AODV) Routing", RFC 3561, July 2003.

[6] J. Haerri, F. Filali, and C. Bonnet, \Performance Demand Distance Vector (AODV) Routing”, RFC 3561, July 2003.
[7] Aamar Nath Muraw et al "performance evaluation of MANET routing protocols GRP, DSR and AODV Based on packet size" IJEST vol. 4 no.06 June 2012.

[8] Laxmi Shrivastava, Sarita S. Bhadauria and G.S. Tomar, "Performance Evaluation of Routing Protocols in MANET with different traffic loads", Proc. of IEEE Conference on Communication Systems and Network Technologies(CSNT 2011).

[9] Sumit Mahajan, Vinay Chopra," Performance Evaluation of MANET Routing Protocols with Scalability using QOS Metrics of VOIP Applications" IJARCSSE Volume 3, Issue 2, February 2013.

[10] Gagangeet Singh Aujla, Sandeep Singh Kang "Comprehensive Evaluation of AODV, DSR, GRP, OLSR and TORA Routing Protocols with varying number of nodes and traffic applications over MANETs "IOSR-JCE Volume 9, Issue 3 (Mar. - Apr. 2013).

[11] Fahim Maan and Nauman Mazhar, "MANET Routing Protocols vs. Mobility Models: A Performance evaluation", Proc. of IEEE-ICUFN 2011.

[12] Gagangeet Singh Aujla, Sandeep Singh Kang," Comprehensive Evaluation of AODV, DSR, GRP, OLSR And TORA Routing Protocols with varying number of Nodes and traffic applications over MANETs" (IOSRJCE).

[13] OPNET official website.

[14] I.S. Hammodi et al, "A comprehensive performance study of OPNET Modeler for ZigBee WSN" 3rd International conference on Next Generation Mobile Applications, 2009. 